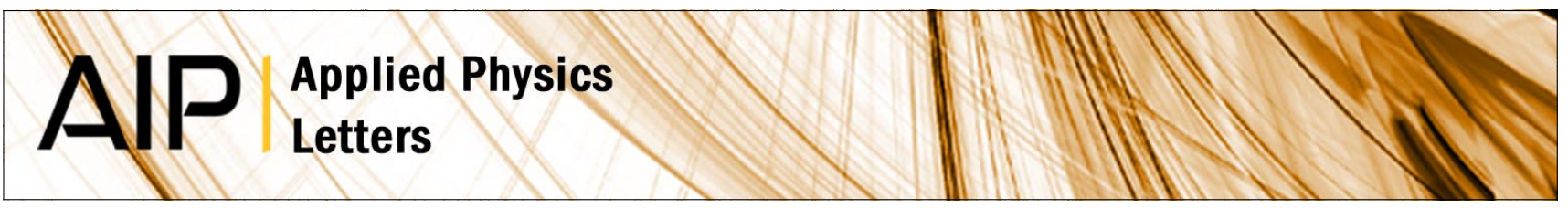

\title{
Optical properties of Mg-doped VO2: Absorption measurements and hybrid functional calculations
}

Shuanglin Hu, S.-Y. Li, R. Ahuja, C. G. Granqvist, K. Hermansson et al.

Citation: Appl. Phys. Lett. 101, 201902 (2012); doi: 10.1063/1.4766167

View online: http://dx.doi.org/10.1063/1.4766167

View Table of Contents: http://apl.aip.org/resource/1/APPLAB/v101/i20

Published by the American Institute of Physics.

\section{Related Articles}

Electronic and optical properties of vacancy-doped WS2 monolayers AlP Advances 2, 042141 (2012)

Effect of N2 dielectric barrier discharge treatment on the composition of very thin SiO2-like films deposited from hexamethyldisiloxane at atmospheric pressure

Appl. Phys. Lett. 101, 194104 (2012)

Ultrafast nonlinear optical responses of bismuth doped silicon-rich silica films

Appl. Phys. Lett. 101, 191106 (2012)

Optical and structural properties of SiOx films grown by molecular beam deposition: Effect of the Si concentration and annealing temperature

J. Appl. Phys. 112, 094316 (2012)

Porosity tuning of the optical properties of mesoporous silica planar defect in macroporous silica opal J. Appl. Phys. 112, 093107 (2012)

\section{Additional information on Appl. Phys. Lett.}

Journal Homepage: http://apl.aip.org/

Journal Information: http://apl.aip.org/about/about_the_journal

Top downloads: http://apl.aip.org/features/most_downloaded

Information for Authors: http://apl.aip.org/authors

\section{ADVERTISEMENT}
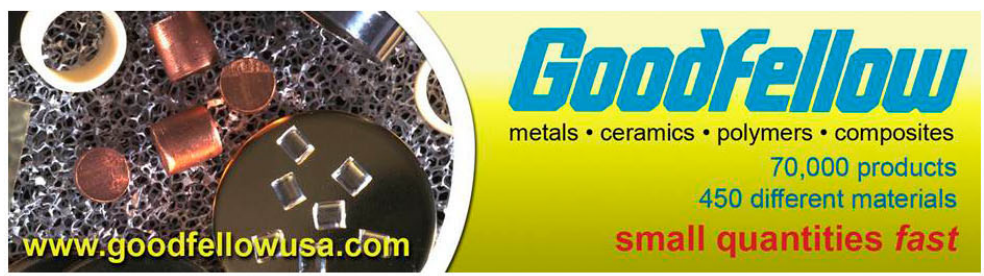


\title{
Optical properties of Mg-doped $\mathrm{VO}_{2}$ : Absorption measurements and hybrid functional calculations
}

\author{
Shuanglin Hu (胡双林), ${ }^{1}$ a) S.-Y. Li (李姝艺), ${ }^{2}$ R. Ahuja, ${ }^{3}$ C. G. Granqvist, ${ }^{2}$ \\ K. Hermansson, ${ }^{1,4}$ G. A. Niklasson, ${ }^{2}$ and R. H. Scheicher ${ }^{3}$ \\ ${ }^{1}$ Department of Chemistry, The Angström Laboratory, Uppsala University, P.O. Box 538, SE-75121 Uppsala, \\ Sweden \\ ${ }^{2}$ Department of Engineering Sciences, The Ångström Laboratory, Uppsala University, P.O. Box 534, \\ SE-75121 Uppsala, Sweden \\ ${ }^{3}$ Department of Physics and Astronomy, The Angström Laboratory, Uppsala University, P.O. Box 516, \\ SE-75120 Uppsala, Sweden \\ ${ }^{4}$ Department of Theoretical Chemistry, The Royal Institute of Technology (KTH), Roslagstullsbacken 15, \\ SE-10691 Stockholm, Sweden
}

(Received 28 September 2012; accepted 22 October 2012; published online 13 November 2012)

\begin{abstract}
$\mathrm{Mg}$-doped $\mathrm{VO}_{2}$ thin films with thermochromic properties were made by reactive DC magnetron co-sputtering onto heated substrates, and spectral absorption was recorded at room temperature in the $0.5<\hbar \omega<3.5 \mathrm{eV}$ energy range. Clear evidence was found for a widening of the main band gap from 1.67 to $2.32 \mathrm{eV}$ as the $\mathrm{Mg} /(\mathrm{V}+\mathrm{Mg})$ atomic ratio went from zero to 0.19 , thereby significantly lowering the luminous absorption. This technologically important effect could be reconciled with spin-polarized density functional theory calculations using the Heyd-Scuseria-Ernzerhof [Heyd et al., J. Chem. Phys. 118, 8207 (2003); ibid. 124, 219906 (2006)] hybrid functional. Specifically, the calculated luminous absorptance decreased when the $\mathrm{Mg} /(\mathrm{V}+\mathrm{Mg})$ ratio was increased from 0.125 to 0.250 . (C) 2012 American Institute of Physics. [http://dx.doi.org/10.1063/1.4766167]
\end{abstract}

This letter reports experimental and theoretical data on $\mathrm{Mg}$-doped $\mathrm{VO}_{2}$ thin films. The main constituent $\left(\mathrm{VO}_{2}\right)$ is a well known thermochromic material capable of reversible transition between a solar-transparent, semiconducting, and monoclinic state below a "critical" temperature $\tau_{c}$ of $\sim 68^{\circ} \mathrm{C}$ and a near-infrared reflecting, metallic-like and tetragonal state above $\tau_{c}{ }^{1,2}$ The associated modulation of solar energy transmittance makes $\mathrm{VO}_{2}$-based thin films a very promising candidate for window coatings for energy efficient buildings. ${ }^{3-6}$ The dopant $(\mathrm{Mg})$ serves to widen the main band gap of $\mathrm{VO}_{2}$ and boosts luminous transmittance, ${ }^{7}$ which otherwise tends to be undesirably low and constitutes a major obstacle for practical implementation of thermochromic fenestration. ${ }^{6} \mathrm{Mg}$ doping also decreases $\tau_{c}$ and brings it closer to room temperature. ${ }^{7} \mathrm{We}$ present spectral measurements of optical absorption in the $0.5<\hbar \omega<3.5 \mathrm{eV}$ range of photon energy and find that these data can be reconciled with hybrid functional calculations.

Mg-doped $\mathrm{VO}_{2}$ thin films were made by reactive DC magnetron sputtering from 5-cm-diameter targets of $\mathrm{V}$ (99.5\%) and $\mathrm{Mg}$ (99.9\%) onto glass and carbon plates concurrently. The substrates were put on a rotating holder located $13 \mathrm{~cm}$ below the targets and were kept at $\sim 450{ }^{\circ} \mathrm{C}$ during depositions. The deposition chamber was evacuated to $6.3 \times 10^{-7}$ mbar before the substrates were heated. $80 \mathrm{ml} / \mathrm{min}$ of $\mathrm{Ar}$ and $5 \mathrm{ml} / \mathrm{min}$ of $\mathrm{O}_{2}$ (both $99.997 \%$ ) were then introduced via mass-flow-controlled inlets. A total pressure of $\sim 9.2$ mTorr was maintained during film fabrication, and sputter powers were $172 \mathrm{~W}$ and $0-40 \mathrm{~W}$ for the $\mathrm{V}$ and $\mathrm{Mg}$ targets, respectively. Films were grown at $\sim 0.056 \mathrm{~nm} / \mathrm{s}$ to thicknesses $d$ of 56 to $67 \mathrm{~nm}$. We recorded $d$ on a Bruker DektakXT profilometer, and supporting data were found by modeling of optical measurements. Elemental

a)Electronic mail: shuanglin.hu@kemi.uu.se. compositions were obtained from Rutherford backscattering spectrometry (RBS) applied to films on carbon. $\mathrm{Mg}$ contents were inferred from simulations based on the SIMNRA program, ${ }^{8}$ which executes iterative least-square fits to experimental spectra. $\mathrm{Mg} /(\mathrm{V}+\mathrm{Mg}) \equiv z$ atomic ratios lay in the $0 \leq z<0.19$ interval.

Spectral normal transmittance $T$ and near-normal reflectance $R$ were recorded in the $0.5<\hbar \omega<3.5 \mathrm{eV}$ range for the semiconducting phase of the films, deposited onto glass, by use of a single-beam spectrophotometer for absolute measurements. ${ }^{9}$ The glass is transparent, and the absorption coefficient $\alpha$ was obtained from Beer-Lambert's Law, i.e., $\alpha d=\ln [T /(1-R)]$. Optical band gaps (denoted $E_{g}$ ) were determined-using standard procedures ${ }^{10}$ - from $(\alpha \hbar \omega)^{m}=A\left(\hbar \omega-E_{g}\right)$, where $A$ is a constant. The exponent $m$ depends on the nature of the optical transition and is $1 / 2$, $1 / 3,2$, and $2 / 3$ for indirect-allowed, indirect-forbidden, directallowed, and direct-forbidden optical transitions, respectively. Linear extrapolation of $(\alpha \hbar \omega)^{m}$ vs $\hbar \omega$ near the band gap gave $E_{g}$ as the intercept with the $\alpha=0$ axis.

Figure 1(a) shows $(\alpha \hbar \omega)^{1 / 2}$ vs $\hbar \omega$ for films with different $\mathrm{Mg}$ contents and presuming indirect-allowed transitions. Extrapolations yield two band gaps for pure $\mathrm{VO}_{2}, E_{g 1}$ and $E_{g 2}$, at $1.67 \mathrm{eV}$ and $\sim 0.5 \mathrm{eV}$, respectively, which are in line with well established data. ${ }^{11}$ It is essential to note that $E_{g 1}$ shifts towards higher energy for increasing doping levels, specifically from $1.67 \mathrm{eV}$ to $2.32 \mathrm{eV}$ as $z$ goes from zero to 0.190 . For $z=0.116$, which corresponds to about one of eight $\mathrm{V}$ atoms substituted by $\mathrm{Mg}$, one finds $E_{g 1} \approx 1.95 \mathrm{eV}$. The strong displacement of $E_{\mathrm{g} 1}$ leads to significantly decreased luminous absorptance in thin films, as inferred from the bell-shaped shaded region in Fig. 1 illustrating the relative luminous efficiency of the eye. ${ }^{12}$

The observation of two band gaps can be rationalized from Goodenough's seminal work on the electronic structure 

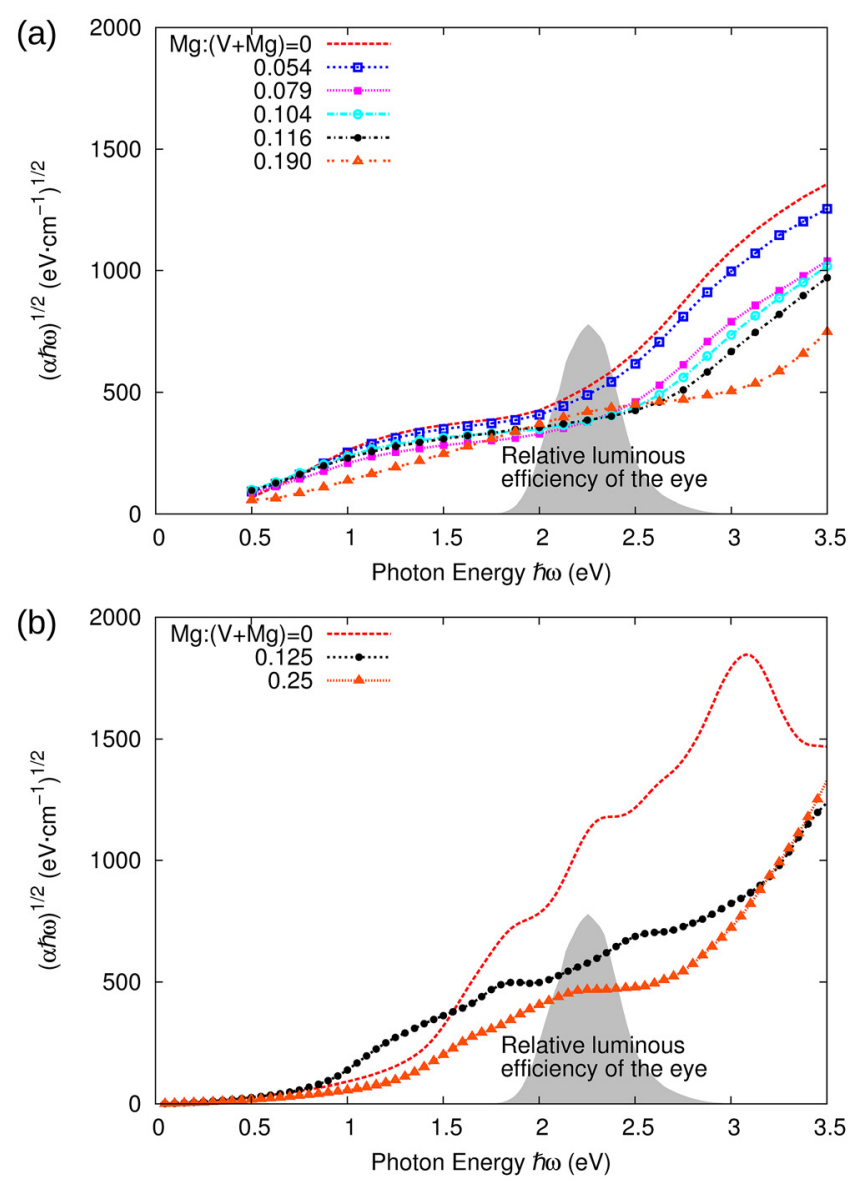

FIG. 1. (a) Experimental and (b) calculated $(\alpha \hbar \omega)^{1 / 2}$ vs photon energy $\hbar \omega$, where $\alpha$ is absorption coefficient, for $\mathrm{Mg}$-doped $\mathrm{VO}_{2}$ with the shown $\mathrm{Mg} /(\mathrm{V}+\mathrm{Mg})$ atomic ratios. The shaded bell-shaped region signifies relative luminous efficiency of the eye (Ref. 12).

of $\mathrm{VO}_{2},{ }^{13}$ which is illustrated in Fig. 2. The crystal-fieldinduced division of the $\mathrm{V} 3 d t_{2 g}$ band into $d_{\|}$and $\pi^{*}$ sub-bands, and further dimerization of $\mathrm{V}$ atoms in the otherwise tetragonal lattice, makes the $d_{\|}$band split into two parts. The lower $d_{\|}$band is completely filled and the $\pi^{*}$ band is empty. $E_{g 1}$ is associated with the band gap between $\mathrm{O} 2 p$ and $\pi^{*}$, and $E_{g 2}$ is associated with the band gap between the lower $d_{\|}$and $\pi^{*}$. This compelling assignment of the band gaps cannot explain the effect of $\mathrm{Mg}$, however, and hence we turn now to calculations.

Electronic and optical properties of the $\mathrm{Mg}$-doped monoclinic $\mathrm{M} 1$ phase of $\mathrm{VO}_{2}$ were studied using the density functional theory (DFT) plane wave basis-set code package

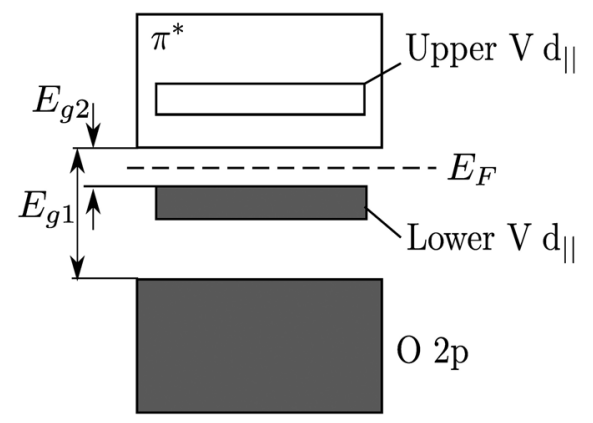

FIG. 2. Schematic band structure for monoclinic, semiconducting $\mathrm{VO}_{2}$ according to Goodenough (Ref. 13). $E_{F}$ denotes Fermi level.
VASP. ${ }^{14}$ Core electrons were treated with the projector augmented wave (PAW) method. ${ }^{15}$ We used the Perdew-BurkeErnzerhof $^{16}$ (PBE) version of the generalized gradient approximation functional and the Heyd-Scuseria-Ernzerhof ${ }^{17}$ (HSE) hybrid functional and performed spin-polarized calculations. The energy cut-off for basis-set expansion was put at $500 \mathrm{eV}$, and the Gaussian broadening width for electronic smearing was $0.05 \mathrm{eV}$. Geometries were relaxed until residual atomic forces were $<0.1 \mathrm{eV} / \mathrm{nm}$, at which point the total energies were calculated to a convergence threshold of $10^{-5} \mathrm{eV}$ per cell for both functionals.

The crystallographic unit cell for the M1 phase comprises four $\mathrm{V}$ and eight $\mathrm{O}$ atoms ${ }^{18}$ and was used in calculations for pure $\mathrm{VO}_{2}$. To simulate $25 \%$ doping, one $\mathrm{V}$ atom was replaced by $\mathrm{Mg}$ in this cell, while $12.5 \%$ doping was simulated by creation of a $2 \times 1 \times 1$ supercell wherein one $\mathrm{V}$ atom was replaced by $\mathrm{Mg}$. Gamma-centered $k$-point grids ${ }^{19}$ of $2 \times 4 \times 4$ (for the supercell) and $4 \times 6 \times 6$ (for the unit cell) were used. The crystal structure-i.e., cell parameters and atomic positions-was relaxed in each case. For pure $\mathrm{VO}_{2}$, this procedure yielded lattice parameters agreeing with experimental data ${ }^{18}$ to within $0 \%-3 \%$ for different cell axes, always exceeding experimental values. $\mathrm{Mg}$ concentrations below $12.5 \%$ would require a supercell at least twice as large, which was deemed too demanding for HSE functional calculations.

The dielectric function $\varepsilon(\omega) \equiv \varepsilon_{1}(\omega)+i \varepsilon_{2}(\omega)$ fully describes the optical properties of a medium at all photon energies. The dielectric function for interband transitions was calculated in the momentum representation, which requires matrix elements between occupied and unoccupied eigenstates. The imaginary part of the dielectric function can be derived from ${ }^{20,21}$

$$
\begin{aligned}
\varepsilon_{2}(\omega)= & \frac{4 \pi^{2} e^{2}}{\Omega} \lim _{q \rightarrow 0} \frac{1}{q^{2}} \sum_{c, v, \mathbf{k}} 2 w_{\mathbf{k}} \delta\left(\epsilon_{c \mathbf{k}}-\epsilon_{v \mathbf{k}}-\omega\right) \\
& \times\left\langle u_{c \mathbf{k}+\boldsymbol{e}_{1} q} \mid u_{v \mathbf{k}}\right\rangle\left\langle u_{c \mathbf{k}+\boldsymbol{e}_{2} q} \mid u_{v \mathbf{k}}\right\rangle^{*},
\end{aligned}
$$

where $e$ is elementary charge, $\Omega$ is volume of the Brillouin zone, indices $c$ and $v$ refer to conduction and valence band states, respectively, $w_{\mathbf{k}}$ is weight of the $k$-point vector, $\epsilon_{c \mathbf{k}}$ and $\epsilon_{v \mathbf{k}}$ are energy levels, $u_{v \mathbf{k}}, u_{c \mathbf{k}+\boldsymbol{e}_{1} q}$, and $u_{c \mathbf{k}+\boldsymbol{e}_{2} q}$ are cell periodic parts of the wavefunction at a given $k$-point, and $e_{1}$ and $e_{2}$ are components of the unit vector. A KramersKroning transformation was used to get the real part $\varepsilon_{1}$ from $\varepsilon_{2}$ by

$$
\varepsilon_{1}(\omega)=1+\frac{2}{\pi} P \int_{0}^{\infty} \frac{\varepsilon_{2}\left(\omega^{\prime}\right) \omega^{\prime}}{\omega^{\prime 2}-\omega^{2}+i \eta} d \omega^{\prime}
$$

where $P$ signifies the principal value and the integral is evaluated in the limit where $\eta$ approaches zero. The absorption coefficient was then derived from

$$
\alpha(\omega)=2(\omega / c)\left\{\left[\left[\varepsilon_{1}^{2}(\omega)+\varepsilon_{2}^{2}(\omega)\right]^{1 / 2}-\varepsilon_{1}(\omega)\right] / 2\right\}^{1 / 2} .
$$

We first consider pure $\mathrm{VO}_{2}$ whose electronic band structure was recently studied with the HSE functional, ${ }^{22,23}$ the 


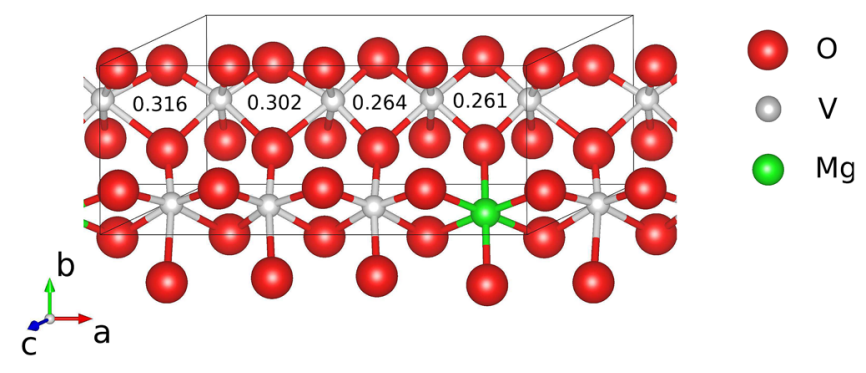

FIG. 3. Optimized structure for $\mathrm{Mg}$-doped $\mathrm{VO}_{2}$ with $\mathrm{Mg} /(\mathrm{V}+\mathrm{Mg})=0.125$, as obtained from calculations using the HSE functional. The figure shows the contents of the $2 \times 1 \times 1$ supercell plus some additional atoms from a neighboring supercell. $\mathrm{V}-\mathrm{V}$ separations are given in nanometers. not aware of any experimental attempts to determine the structural modifications invoked in $\mathrm{VO}_{2}$ by $\mathrm{Mg}$ doping.

We also studied the electronic properties of Mg-doped $\mathrm{VO}_{2}$, and Fig. 4 shows density of states for $z=0.125$ and $z=0.25$. The overall agreement with Goodenough's band scheme, ${ }^{13}$ illustrated in Fig. 2, is apparent. The band gaps $E_{g 1}$ and $E_{g 2}$, calculated from the eigenvalues, are noted in the figure. Specifically, our $E_{g 1}$ and $E_{g 2}$ values are 2.07 and $0.72 \mathrm{eV}$ for $z=0.125$ and 2.12 and $1.01 \mathrm{eV}$ for $z=0.25$, respectively. Band structure calculations give clear evidence of indirect band gaps for $z=0.25$ and pure $\mathrm{VO}_{2}$.

Summarizing, we investigated the optical properties of Mg-doped $\mathrm{VO}_{2}$ films experimentally and computationally.
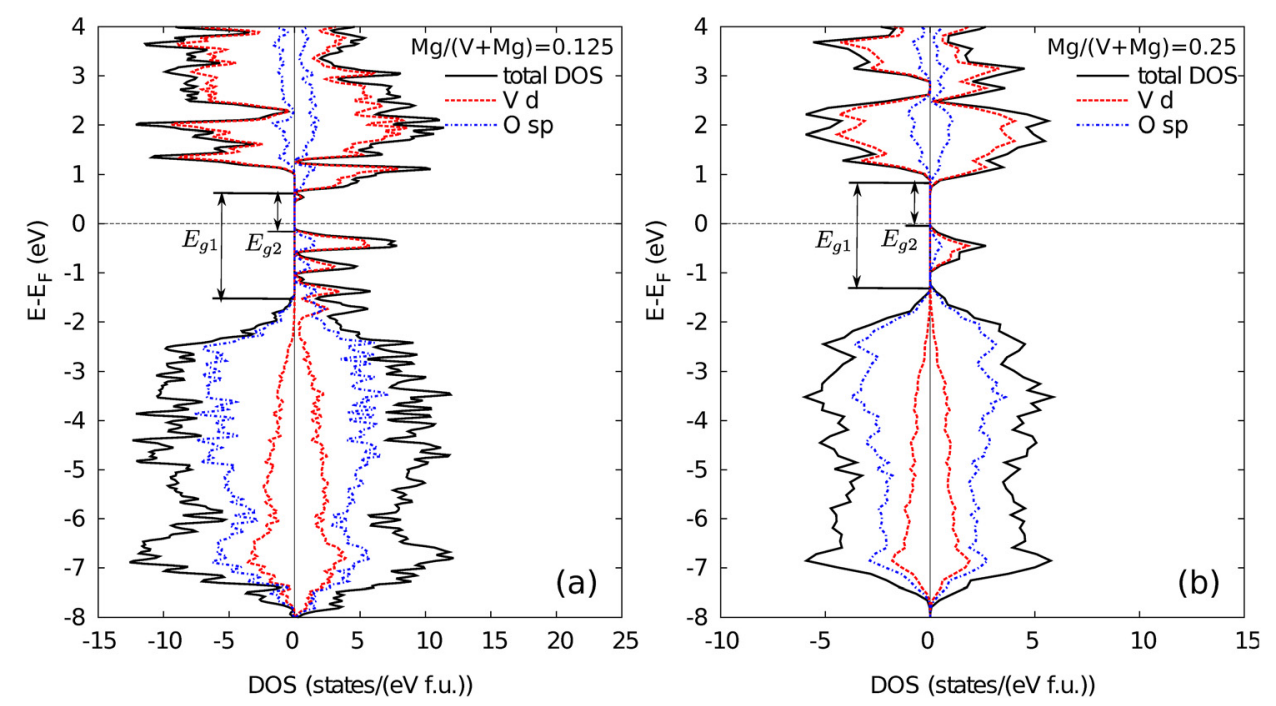

FIG. 4. Total and partial density of states (DOS) for $\mathrm{V}$ and $\mathrm{O}$ calculated by use of the HSE functional for $\mathrm{Mg}$-doped $\mathrm{VO}_{2}$ with the $\mathrm{Mg} /(\mathrm{V}+\mathrm{Mg})$ atomic ratio (a) 0.125 and (b) 0.25 . Characters of the bands are noted, as are the band gaps. $E_{F}$ denotes Fermi energy and f.u. means formula unit. electronic and optical properties have also been calculated by several other methods. ${ }^{24}$ Our results are almost identical to those of Eyert. ${ }^{22}$ Specifically, we find an indirect band gap of $1.38 \mathrm{eV}$ corresponding to the difference between the maximum and the minimum of the bands in $k$-space. Figure 1(b) shows calculated spectral absorption coefficients for $\mathrm{VO}_{2}$ as well as the doped systems. A comparison with the experimental data in Fig. 1(a) for $z=0.116$ and $z=0.190$-which are the compositions closest to those of the calculationsshows clear qualitative correspondence with theory.

The match between experimental and calculated spectral absorption is better for the Mg-doped samples than for pure $\mathrm{VO}_{2}$, and one may ask why this is so. We speculate that a possible answer could be that electron correlation effects in $\mathrm{VO}_{2}$ are reduced as a consequence of the $\mathrm{Mg}$ doping, thus allowing a more accurate description of electronic structure with DFT-based methods. Substitution of some V by $\mathrm{Mg}$ clearly influences the structure and leads to rearrangement of ionic positions, notably those of $\mathrm{V}$. Thus, our calculations predict that the alternating $\mathrm{V}-\mathrm{V}$ distances $(0.315$ and $0.252 \mathrm{~nm}$, etc.) for pure $\mathrm{VO}_{2}$ along the $\mathrm{V}$ chain in the $a$ direction (rutile $c$ axis) change for $z=0.125$ and, moreover, become pair-wise similar $(0.316$ and $0.302 \mathrm{~nm} ; 0.264$ and $0.261 \mathrm{~nm}$, etc.), as indicated in Fig. 3. It is interesting to note that even this modest $\mathrm{Mg}$ content affects the structure throughout the supercell. For $z=0.25$, the $\mathrm{V}-\mathrm{V}$ distances along the $a$ direction become 0.302 and $0.290 \mathrm{~nm}$. We are
The qualitative agreement between the two sets of data gives strong evidence that the band gap widening - and concomitantly the technically important lowering of the luminous absorptance-can be reconciled with hybrid functional calculations. This finding may also open avenues towards computation to discover alternative band gap widening dopants for thermochromic and nano-thermochromic ${ }^{25} \mathrm{VO}_{2}$ based window coatings. Furthermore, our work may be relevant to other potential applications of $\mathrm{VO}_{2}$-based materials, such as nanoelectronic switches ${ }^{26}$ and microbolometers. ${ }^{27}$

R.A., C.G.G., K.H., G.A.N., and R.H.S. acknowledge financial support from the Swedish Research Council (VR). The calculations were performed by use of resources provided by the Swedish National Infrastructure for Computing (SNIC) at UPPMAX and NSC. We are grateful to A. Hallén, P. C. Lansåker, P. Petersson, and G. Possnert for RBS measurements and helpful discussions on materials analysis.

${ }^{1}$ F. J. Morin, Phys. Rev. Lett. 3, 34 (1959).

${ }^{2}$ S. M. Babulanam, T. S. Eriksson, G. A. Niklasson, and C. G. Granqvist, Sol. Energy Mater. 16, 347 (1987).

${ }^{3}$ G. B. Smith and C. G. Granqvist, Green Nanotechnology: Solutions for Sustainability and Energy in the Built Environment (CRC, Boca Raton, FL, 2010).

${ }^{4}$ M. Saeli, C. Piccirillo, I. P. Parkin, R. Binions, and I. Ridley, Energy Build. 42, 1666 (2010).

${ }^{5}$ Y. Gao, H. Luo, Z. Zhang, L. Kang, Z. Chen, J. Du, M. Kanehira, and C. Cao, Nano Energy 1, 221 (2012). 
${ }^{6}$ S.-Y. Li, G. A. Niklasson, and C. G. Granqvist, Thin Solid Films 520, 3823 (2012).

${ }^{7}$ N. R. Mlyuka, G. A. Niklasson, and C. G. Granqvist, Appl. Phys. Lett. 95, 171909 (2009).

${ }^{8}$ M. Mayer, AIP Conf. Proc. 475, 541 (1999).

${ }^{9}$ P. Nostell, A. Roos, and D. Rönnow, Rev. Sci. Instrum. 70, 2481 (1999).

${ }^{10} \mathrm{~F}$. Wooten, Optical Properties of Solids (Academic, New York, 1972).

${ }^{11}$ H. W. Verleur, A. S. Barker, Jr., and C. N. Berglund, Phys. Rev. 172, 788 (1968); H. S. Choi, J. S. Ahn, J. H. Jung, T. W. Noh, and D. H. Kim, Phys. Rev. B 54, 4621 (1996); K. Okazaki, S. Sugai, Y. Muraoka, and Z. Hiroi, ibid. 73, 165116 (2006); M. M. Qazilbash, K. S. Burch, D. Whisler, D. Shrekenhamer, B. G. Chae, H. T. Kim, and D. N. Basov, ibid. 74, 205118 (2006); J. B. Kana Kana, J. M. Ndjaka, C. Vignaud, A. Gibaud, and M. Maaza, Opt. Commun. 284, 807 (2011); W. W. Li, J. J. Zhu, X. F. Xu, K. Jiang, Z. G. Hu, M. Zhu, and J. H. Chu, J. Appl. Phys. 110, 013504 (2011). Specifically, $E_{g 1}$ is in excellent agreement with recent observations for $\mathrm{VO}_{2}$ films made by sputtering of vanadium followed by post oxidation [Z. Luo, Z. Wu, X. Xu, T. Wang, and Y. Jiang, J. Vac. Sci. Technol. A 28, 595 (2010); Chin. Phys. B 19, 106103 (2010)]; it is also consistent with band gaps for some sol-gel-prepared $\mathrm{VO}_{2}$ films [M. M. Qazilbash, A. A. Schafgans, K. S. Burch, S. J. Yun, B. G. Chae, B. J. Kim, H. T. Kim, and D. N. Basov, Phys. Rev. B 77, 115121 (2008)], bcc-type $\mathrm{VO}_{2}$ nanorod arrays [Y. Q. Wang, Z. J. Zhang, Y. Zhu, Z. C. Li, R. Vajtai, L. J. Ci, and P. M. Ajayan, ACS Nano 2, 1492 (2008)], and over-stoichiometric vanadium surfaces [A. Z. Moshfegh and A. Ignatiev, Thin Solid Films 198, 251 (1991)]. Band gaps evaluated from $(\alpha \hbar \omega)^{2}$ vs $\hbar \omega$ - as would be adequate for direct-allowed transitions-may appear as much as $\sim 1 \mathrm{eV}$ wider than if the assessment is for indirect-allowed transitions.

${ }^{12}$ G. Wyszecki and W. S. Stiles, Color Science: Concepts and Methods, Quantitative Data and Formulae, 2nd ed. (Wiley, New York, 2000).
${ }^{13}$ J. B. Goodenough, J. Solid State Chem. 3, 490 (1971).

${ }^{14}$ G. Kresse and J. Hafner, Phys. Rev. B 48, 13115 (1993); G. Kresse and J. Furthmüller, ibid. 54, 11169 (1996); G. Kresse and D. Joubert, ibid.. 59, 1758 (1999).

${ }^{15}$ P. E. Blöchl, Phys. Rev. B 50, 17953 (1994).

${ }^{16}$ J. P. Perdew, K. Burke, and M. Ernzerhof, Phys. Rev. Lett. 77, 3865 (1996); erratum 78, 1396 (1997).

${ }^{17}$ J. Heyd, G. E. Scuseria, and M. Ernzerhof, J. Chem. Phys. 118, 8207 (2003); erratum 124, 219906 (2006).

${ }^{18}$ G. Andersson, Acta Chem. Scand. 10, 623 (1956).

${ }^{19}$ H. J. Monkhorst and J. D. Pack, Phys. Rev. B 13, 5188 (1976).

${ }^{20}$ R. Ahuja, S. Auluck, J. M. Wills, M. Alouani, B. Johansson, and O. Eriksson, Phys. Rev. B 55, 4999 (1997).

${ }^{21}$ M. Gajdoš, K. Hummer, G. Kresse, J. Furthmüller, and F. Bechstedt, Phys. Rev. B 73, 045112 (2006).

${ }^{22}$ V. Eyert, Phys. Rev. Lett. 107, 016401 (2011).

${ }^{23}$ F. Iori, M. Gatti, and A. Rubio, Phys. Rev. B 85, 115129 (2012)

${ }^{24}$ A. Liebsch, H. Ishida, and G. Biehlmayer, Phys. Rev. B 71, 085109 (2005); M. Gatti, F. Bruneval, V. Olevano, and L. Reining, Phys. Rev. Lett. 99, 266402 (2007); J. M. Tomczak and S. Biermann, Phys. Rev. B 80, 085117 (2009); Phys. Status Solidi B 246, 1996 (2009); Europhys. Lett. 86, 37004 (2009); R. Su, J. He, and Y.-J. Guo, Acta Phys. Sin. 60, 107101 (2011); A. S. Belozerov, M. A. Korotin, V. I. Anisimov, and A. I. Poteryaev, Phys. Rev. B 85, 045109 (2012).

${ }^{25}$ S.-Y. Li, G. A. Niklasson, and C. G. Granqvist, J. Appl. Phys. 108, 063525 (2010); 109, 113515 (2011); Appl. Phys. Lett. 99, 131907 (2011).

${ }^{26}$ M. Nakano, K. Shibuya, D. Okuyama, T. Hatano, S. Ono, M. Kawasaki, Y. Iwasa, and Y. Tokura, Nature 487, 459 (2012).

${ }^{27}$ L. A. L. de Almeida, G. S. Deep, A. M. N. Lima, I. A. Khrebtov, V. G. Malyarov, and H. Neff, Appl. Phys. Lett. 85, 3605 (2004). 